\title{
Nucleolar GTPase NOG-1 Regulates Development, Fat Storage, and Longevity through Insulin/IGF Signaling in C. elegans
}

\author{
Young-II Kim ${ }^{2,5}$, Jaya Bandyopadhyay, ${ }^{3,5}$, Injeong Cho ${ }^{1}$, Juyeon Lee ${ }^{4}$, Dae Ho Park ${ }^{4}$, and \\ Jeong Hoon $\mathrm{Cho}^{1, *}$
}

NOG1 is a nucleolar GTPase that is critical for 605 ribosome biogenesis. Recently, NOG1 was identified as one of the downstream regulators of target of rapamycin (TOR) in yeast. It is reported that TOR is involved in regulating lifespan and fat storage in Caenorhabditis elegans. Here, we show that the nog1 ortholog (T07A9.9: nog-1) in C. elegans regulates growth, development, lifespan, and fat metabolism. A green fluorescence protein (GFP) promoter assay revealed ubiquitous expression of $C$. elegans nog-1 from the early embryonic to the adult stage. Furthermore, the GFP-tagged NOG-1 protein is localized to the nucleus, whereas the aberrant NOG-1 protein is concentrated in the nucleolus. Functional studies of NOG-1 in C. elegans further revealed that nog-1 knockdown resulted in smaller broodsize, slower growth, increased life span, and more fat storage. Moreover, nog-1 over-expression resulted in decreased life span. Taken together, our data suggest that nog-1 in C. elegans may be an important player in regulating life span and fat storage via the insulin/IGF pathway.

\section{INTRODUCTION}

Small guanine triphosphate (GTP) ases are well known regulators of diverse cellular and developmental events such as differentiation, cell cycle regulation, vesicle trafficking, microtubule organization, and ribosome assembly via GTP-GDP conformational changes. Caenorhabditis elegans contains approximately

${ }^{1}$ Department of Biology Education, College of Education, Chosun University, Gwangju 501-759, Korea, ${ }^{2}$ Biomedical Research Center, Korea Advanced Institute of Science and Technology, Daejeon 305-701, Korea, ${ }^{3}$ Department of Biotechnology, West Bengal University of Technology, Salt Lake City, Kolkata 700-064, India, ${ }^{4}$ Department of Life Science, Gwangju Institute of Science and Technology, Gwangju 500-712, Korea, ${ }^{5}$ These authors contributed equally to this work.

*Correspondence: renocho@ chosun.ac.kr

Received 9 September, 2013; revised 28 November, 2013; accepted 29 November, 2013; published online 27 January, 2014

Keywords: $C$. elegans, daf-2, daf-16, fat metabolism, insulin/IGF-1, life span, NOG-1, nog-1, nucleolar GTPase, r $r p-24.2$
50 Ras GTPase subfamily genes, including genes of the Ras/Rap/Ral, Rho, Rab, Arf/Sar, and Ran families (Lundquist 2006). ran-1, one of the three ran genes in $C$. elegans, is a well-characterized nuclear GTPase gene, and functions in nuclear trafficking, nuclear reassembly, and kinetochore association with the mitotic spindle (Askjaer et al., 2002).

Ran is a typical nuclear GTPase that regulates the transportation of transfer RNA, ribosomal subunits, and nuclear localization sequence (NLS) and nuclear export signal (NES) proteins from the cytosol to the nucleus and vice versa. It may directly or indirectly control these transfers through importin, exportin, GTPase activating proteins (GAPs), and guanine exchange factors (GEFs) (Kutay et al., 1998; Moy and Silver, 1999). More recent reports indicate that Ran may be involved in diverse cytoplasmic events, such as trafficking an ephrin receptor homolog in nematode oocytes, controlling neurite outgrowth in Drosophila and in mammalian neurons, and retrograde signaling in nerve axons after injury (Yudin and Fainzilber, 2009).

NOG1, a nuclear GTP-binding protein, plays a significant role during the later stages of ribosome biogenesis, particularly in $60 S$ ribosome biogenesis (Jensen et al., 2003). Moreover, NOG1 is highly conserved from yeast to humans, which indicates its essential role in cell viability (Honma et al., 2006). According to the results of a proteome-wide project, NOG1 is localized to the nucleus, where it acts as a critical regulator for ribosome maturation in response to nutrient availability (Honma et al., 2006; Huh et al., 2003; Jensen et al., 2005). Ribosomal RNAs (rRNAs) and ribosomal proteins (RPs) are synthesized, assembled, and experience maturation during ribosome biosynthesis (Kressler et al., 1999; Lafontaine and Tollervey, 2001; Venema and Tollervey, 1999). In addition to rRNAs and RPs, several other nuclear and nucleolar proteins are known to participate in ribosome assembly pathways (Fromont-Racine et al., 2003; Tschochner and Hurt, 2003).

Ribosome biogenesis is a highly energy-dependent process related to nutrient availability, and the process is regulated by target of rapamycin (TOR) (Powers et al., 1999). TOR upregulates the transcriptions of rRNAs and mRNAs of RPs in yeast and mammals (Claypool et al., 2004; Hannan et al., 2003; Mayer and Grummt, 2006; Tsang et al., 2003). Moreover, TOR activity is required for the early and late stages of ribosome maturation in the nucleoplasm and for rRNA processing. Interestingly, TOR is potentially a downstream component in the 
insulin/insulin-like growth factor (IGF) signaling pathway that is essential for growth and body size (Oldham and Hafen, 2003). TOR may interact directly or indirectly with insulin/IGF signaling in order to regulate a common set of proteins that are involved in the control of cell growth, such as translation initiation factor 4E-binding protein (4EBP1) and S6 ribosomal protein kinase (Schmelzle and Hall, 2000).

Recently, deletion of genes that either encode $60 \mathrm{~S}$ subunit proteins or processing factors have been shown to significantly increase yeast lifespan. Moreover, treatment alone of $60 \mathrm{~S}$ subunit biogenesis inhibitors increases lifespan sufficiently (Steffen et al., 2008). Furthermore, C. elegans with reduced levels of ribosomal protein S6 (translation initiation factor) and some ribosomal subunits showed an increase in longevity, which may be the result of increased stress resistance (Hansen et al., 2007; Pan et al., 2007). Therefore, it is postulated that NOG1 might modulate longevity via ribosomal biogenesis regulation.

Insulin/IGF signaling regulates larval development and adult life span in C. elegans (Kenyon et al., 1993; Kimura et al., 1997). In addition, the TOR pathway in $C$. elegans is known to interact with the insulin signaling pathway to regulate larval development, metabolism, and life span (Jia et al., 2004). TOR was also found to be involved in controlling ribosome biogenesis via NOG1 in Saccharomyces cerevisiae (Honma et al., 2006). In C. elegans, T07A9.9 was identified as a novel ortholog of NOG1 (hereafter NOG-1). Therefore, we hypothesize that in $C$. elegans, nucleolar GTPase NOG-1 regulates development and life span through the insulin/IGF signaling pathway and/or the TOR signaling pathway. Our results suggest a plausible genetic interaction of nog-1 with both insulin/IGF and TOR signaling. Furthermore, NOG-1 may play an important role in development and longevity in $C$. elegans.

\section{MATERIALS AND METHODS}

\section{Strains and culture conditions}

The wild-type Bristol N2, daf-16(mu86), sir-2.1(ok434) were obtained from the Caenorhabditis Genetics Center (CGC) at the University of Minnesota. The maintenance of $C$. elegans was carried out according to protocols from Brenner (1974).

\section{Plasmids and expression studies}

Approximately $1.0 \mathrm{~kb}$ of the $5^{\prime}$ upstream region (promoter) of nog-1 (T07A9.9) was amplified by polymerase chain reaction (PCR), and worm lysate was used as the template. The amplified DNA was cloned into a promoterless green fluorescent protein (GFP) vector, pPD95.77, to generate the nog-1promoter: gfp (Pnog-1::gfp) construct. For translation fusion constructs, nog-1 cDNA was amplified by reverse transcription (RT)-PCR and standard PCR techniques and cloned into the same vector, nog-1 promoter::nog-1::gfp (Pnog-1::nog-1::gfp). A nog -1 G225A construct was obtained by a site-directed mutation using onestep overlap extension PCR (Urban et al., 1997).

Microinjection was carried out as previously described (Mello and Fire, 1995). As a transformation marker, a plasmid pRF4 containing a dominant gene (rol-6) was co-injected. The transgenic worms were isolated by their rolling pattern and GFP expressions using a NIKON fluorescent stereomicroscope SMZ1000. Images were captured using a Nikon microscope Eclipse 80i-DS-Fi1 system.

For RNAi bacterial feeding constructs, target cDNAs ( $g f p$, daf-2, daf-16, nog-1, and rlp-24.2) were obtained by RT-PCR and standard PCR techniques, and the cDNAs were cloned into the L4440 Fire vector for bacteria-feeding-mediated RNAi.
The RNAi constructs were eventually transformed and maintained in HT115 (DE3) Escherichia coli hosts.

nog-1 and rlp-24.2 over-expression constructs were generated using a specific heat shock promoter containing vectors (pPD49.83). nog-1 and rlp-24.2 cDNAs were amplified by RTPCR and standard PCR, and then sub-cloned into pPD49.83 vectors. Following microinjections of these specified constructs, transgenic worms or their eggs were heat shocked at $30^{\circ} \mathrm{C}$ for $1 \mathrm{~h}$ over a $12-\mathrm{h}$ interval.

\section{RNA-mediated interference}

A single colony of the RNAi construct-transformed bacteria was incubated overnight in Luria-Bertani (LB) broth with $100 \mu \mathrm{g} / \mathrm{ml}$ ampicillin. The next day, after the culture was diluted and grown for another $6 \mathrm{~h}$, it was spread onto Nematode Growth Medium (NGM) plates containing $50 \mu \mathrm{g} / \mathrm{ml}$ ampicillin and $1 \mathrm{mM}$ isopropyl-1-thio- $\beta$-D-galactopyranoside (IPTG). The plates were grown overnight at $20^{\circ} \mathrm{C}$, allowing for the growth of a lawn of $E$. coli that expressed double-stranded RNAs (dsRNAs) of the target gene. Once ready, synchronized L4 worms were transferred and changed to fresh RNAi plates at 2-day intervals.

\section{Phenotype analysis}

The brood sizes and lifespans of gfp, daf-2, daf-16, nog-1, and rlp-24.2 RNAi-treated worms were scored at $20^{\circ} \mathrm{C}$. Individual worms were placed on RNAi bacterial feeding plates and allowed to self-fertilize at $20^{\circ} \mathrm{C}$. The PO worms were transferred to fresh plates at 12-h intervals for 4 subsequent days, and the number of progeny $(\mathrm{F} 1)$ was counted. The life spans of the worms were assayed at $20^{\circ} \mathrm{C}$. The $\mathrm{L} 4$ stage hermaphrodites were transferred to fresh plates daily or at 2-day intervals until they stopped laying eggs. If the worms failed to move when prodded or if they lacked pharyngeal pumping, the worms were scored as dead. If a worm crawled off the plate, it was excluded from the assay. The analyses were repeated three times. Statistical analyses were conducted using the software OASIS (Yang et al., 2011).

\section{Sudan Black B staining}

L4 stage C. elegans were exposed to RNAi (gfp, daf-2, daf-16, and nog-1) feeding plates. After 5 days, the worms were removed from the plates and subsequently starved for $6 \mathrm{~h}$. Following washing in M9, the worms were fixed with $1 \%$ paraformamide in M9. The worms were subjected to three freeze-thaw cycles and then dehydrated in $70 \%$ ethanol. For staining experiments, Sudan Black B stain was saturated in $70 \%$ ethanol and then added to the dehydrated worm samples. The worms were incubated overnight under mild shaking at room temperature followed by washing in $70 \%$ ethanol 4 times to eliminate any nonspecific dye. The supernatant was removed carefully followed by the addition of M9 buffer.

\section{RESULTS AND DISCUSSION}

\section{C. elegans nog- 1 is an evolutionary conserved GTP-binding GTPase}

Several GTP-binding proteins are well known for their critical roles in the signaling cascades of growth factors and hormones affecting the development of mammalian renal cells (Laping et al., 2001). It was also recently discovered that the chronic renal failure gene (CRFG) encodes a novel GTP-binding protein that expresses in the outer medulla of the kidney. CRFG products in humans and rats are known as orthologs of NOG-1. However, C. elegans nog-1 has so far not been identified. Therefore, we 


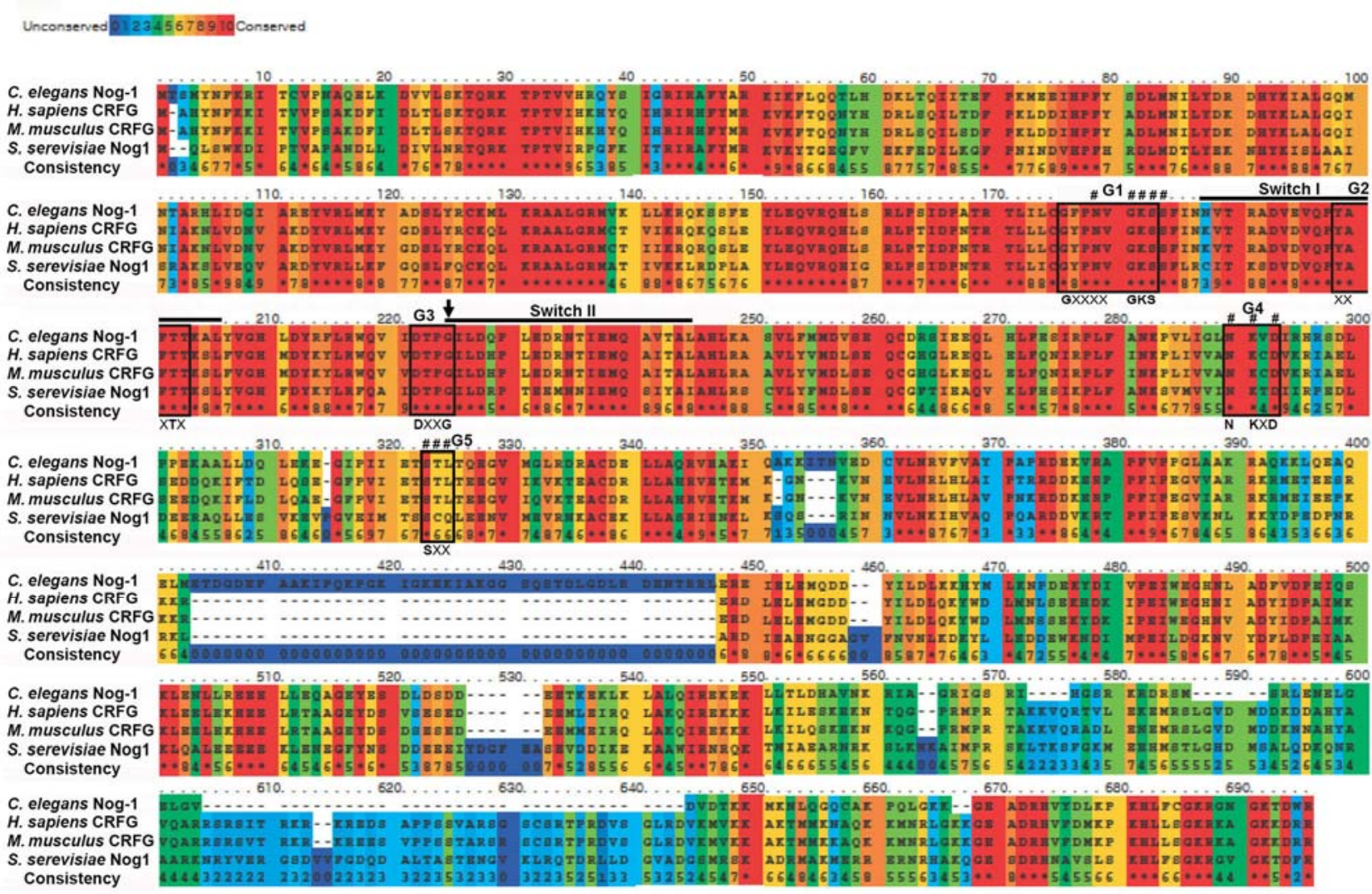

Fig. 1. C. elegans NOG-1 is a conserved protein. Amino acid sequence alignment of NOG-1 among $C$. elegans (C. elegans NOG-1; accession no. O44411), human CRFG ( $H$. sapiens CRFG; accession no. Q9BZE4), mouse CRFG ( $M$. musculus CRFG; accession no. Q99ME9), and yeast NOG1(S. cerevisiae NOG1, accession no. Q02892) by PRALINE program. Sequence similarities among NOG-1/CRFG homologues are represented by different colored boxes (from red to blue, high conserved to less conserved). Black box, G1 to G5 boxes; black upper line, Switch I and Switch II region; \#, GTP/Mg ${ }^{2+}$ binding; black arrow, G225A (G224A in mouse).

compared candidates for NOG-1 (T07A9.9: nog-1) in C. elegans with other NOG1 proteins or homologs in humans, mice, and yeast. NOG1 belongs to the obg-like family of GTPases, which includes Obg, DRG, YyaF/Ychf, and Ygr210 (MarchlerBauer et al., 2013). The Obg-like family of GTPases contains domains of GTP/Mg ${ }^{2+}$, Switch I, Switch II, and G1-G5 boxes. The GTP/Mg ${ }^{2+}$ binding domain is essential for GTPase activity (Buglino et al., 2002), whereas Switch I and overlapping G2 box are essential effector sites (Buglino et al., 2002; Fuentes et al., 2007). The alignment showed that NOG-1-specific domains are all conserved across the different animal groups (Fig. 1). In addition, G2-specific threonine residues are also well conserved in these organisms (Fig. 1; Fuentes et al., 2007). C. elegans NOG-1 shows $54.7 \%$ identity with human CRFG, $54 \%$ identity with mouse CRFG, and $42.3 \%$ identity with yeast NOG1. Given the high similarity with mammalian CRFGs and yeast NOG1, NOG-1 maybe a functionally conserved protein.

Expression and localization of $C_{\text {. elegans nog-1 }}$

To examine spatial and temporal expression patterns of NOG-1, plasmids of GFP transcriptional fusion (Pnog-1::gfp, Figs. 2A$2 \mathrm{H}$ ) or GFP translational fusion (Pnog-1::nog-1::gfp; Figs. 2l$2 \mathrm{~K}$ ) were constructed and subsequently injected into wild-type young adults (N2 background). Analyses of the transgenic worms revealed that Pnog-1::gfp fluorescence was ubiquitous.
Moreover, the expression was distinct throughout all stages of development, from early embryos to adult stages (Figs. 2A-2C). Green fluorescence expression was also prominent in the intestine (Fig. 2D), germ cells (Fig. 2E), body wall muscles (Fig. 2F), pharynx, and excretory cells (Fig. 2G). NOG-1 was expressed in the vulva and neurons as well (Fig. $2 \mathrm{H}$ ). This ubiquitous expression pattern may implicate a wide range of NOG-1 functions in the nematode. For further investigation, translational GFP (Pnog-1::nog-1::gfp) expression and nucleus staining (DAPI) experiments were performed. In intestinal cells, NOG-1 GFP accumulated specifically in the nucleolus (Figs. 2l and 2J).

The GTP binding core ( $\mathrm{G}$ domain) of GTPase consists of signature motifs (G1-G5) that are vital for GTP-binding and their functions. Conserved residues within the $\mathrm{G}$ domain of the mouse nog1 gene were mutated. Among the mutants, a substitution of alanine for glycine in the Switch II segment of NOG1 (G224A) showed a prominent phenotype of cell growth inhibition. The G224A mutant displayed localized NOG1 accumulation in the nucleolus (Lapik et al., 2007). Therefore, NOG-1 localization in $C$. elegans was performed using transgenic worms containing GFP translational fusion constructs that carried the NOG-1 GTP binding site defective mutation [Pnog-1::nog-1 (G225A):: $g f p]$. Analyses of the transgenics showed aggregated expression patterns of GFP (Fig. 2K) in contrast to those of wild-type expression patterns (Fig. 2J). 

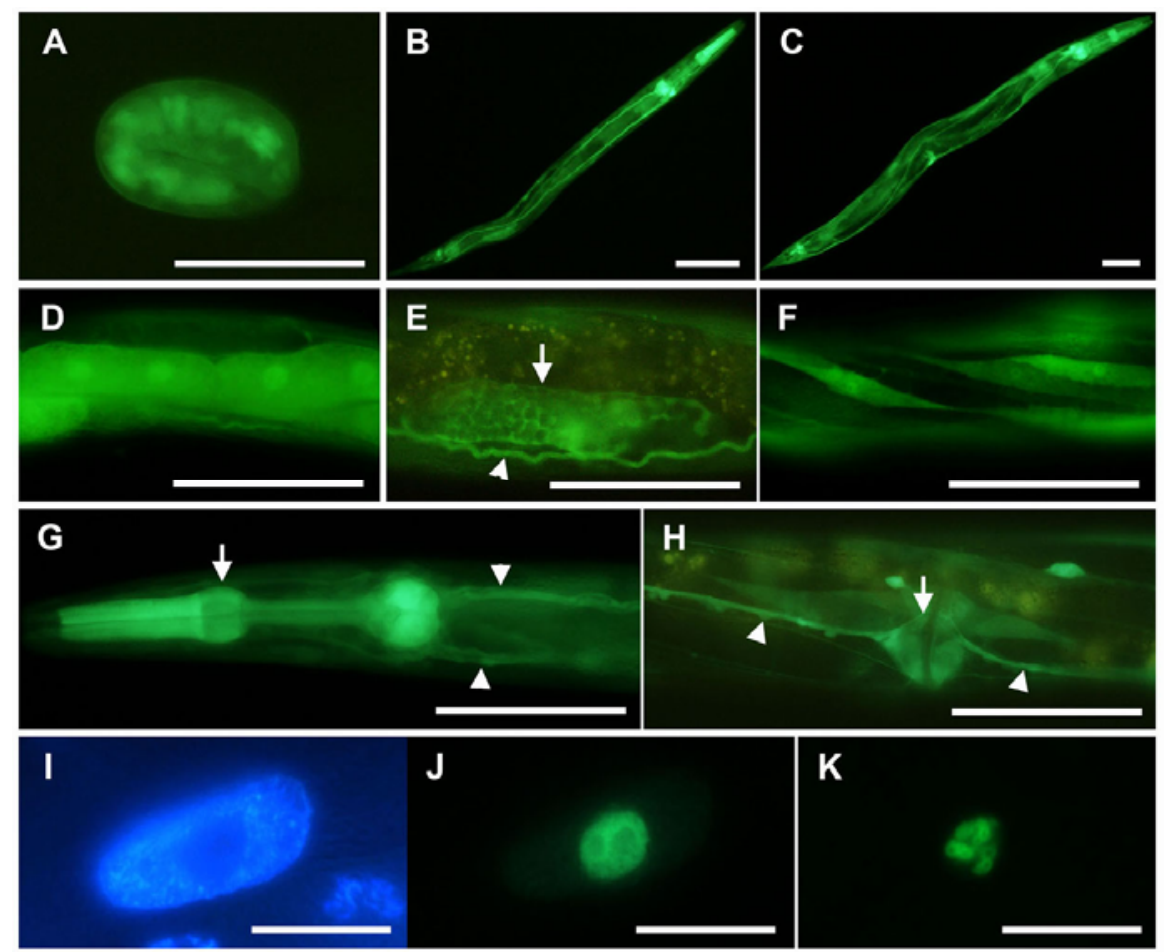

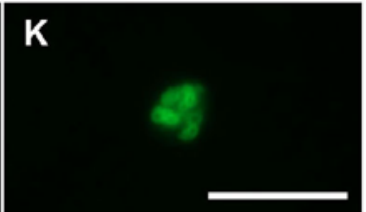

Fig. 2. Expression and localization of C. elegans nog-1. GFP transcriptional fusion constructs (Pnog-1::gfp) and NOG-1 GFP translational fusion constructs (Pnog-1::nog-1::gfp) were injectted into wild-type young adults. GFP expressions were examined and photographed in the transgenic worms under a fluorescence microscope. Pnog1:: gfp fluorescence was expressed from early embryo stages to adult stages (A, embryo; B, larva stage; C, adult). The expression was observed in intestine (D), germ cells (arrow) and excretory cells (arrowhead) (E), body wall muscles $(F)$, in pharynx (arrow) and excretory cells (arrowhead) (G) and in vulva (arrow), and neurons [arrowhead, $(\mathrm{H})$ ]. Nucleus NOG-1::GFP localization in intestine cells; DAPI staining (I), NOG-1::GFP localization in nucleolus (J), and NOG-1G225A (GTP binding site defect) localization in nucleolus (K). (I) and $(\mathrm{J})$ are obtained from the same cell. Scale bar, (A-H), $50 \mu \mathrm{m}$; (I-K), $10 \mu \mathrm{m}$.
Moreover, G224A ectopic expression showed disruption of pre-60S assembly and arrest of cell proliferation (Lapik et al., 2007). The precise glycine position of NOG-1 is required for the accurate maintenance of their molecular structure and proper conformational transition upon GTP-GDP exchanges. Our GFP translational expression data with a dominant-negative mutation further demonstrated the importance the glycine residue in NOG-1 in $C$. elegans and are consistent with the mammalian reports.

nog-1 knockdown in C. elegans shows decreased brood size and growth rates

To investigate nog-1 function in vivo, control (gfp), daf-2, daf-16, and nog-1 (for knockdown effect) RNAi bacteria were fed to wild type $C$. elegans. The brood sizes and growth rates of treated worms were examined (Figs. $3 \mathrm{~A}$ and $3 \mathrm{~B}$ ). Interestingly, the nog-1 RNAi worms displayed an extremely reduced mean brood size ( $31.80 \pm 17.37, n=5$ trials) in comparison to the control RNAi worms $(244.2 \pm 35.10, n=5$ trials), daf-2 RNAi worms (200.6 $\pm 21.82, \mathrm{n}=5$ trials), and daf-16 RNAi worms (182.0 $\pm 16.94, n=5$ trials) (Fig. $3 A$ ). In addition, the growth rate of nog-1 knockdown worms was significantly slower than that of any of the other RNAi worms; the average time to reach adulthood in nog-1 RNAi worms was approximately $90.06 \mathrm{~h}$, whereas control RNAi worms reached adulthood at an average of $49.85 \mathrm{~h}$ (Fig. 3B). These results suggest that nog-1 is required for normal brood size and growth in $C$. elegans. Furthermore, it is plausible that the extremely slow growth of the nog-1 RNAi worms may affect the brood size.

NOG-1 regulates life span through the insulin/IGF-1 pathway

It is known that insulin/IGF signaling regulates larval develop- ment and adult life span in C. elegans (Kenyon et al., 1993; Kimura et al., 1997). In addition, the target of the rapamycin (TOR) pathway interacts with the insulin signaling pathway to regulate the development, metabolism, and life span of $C$. elegans (Jia et al., 2004). The NOG-1 protein is also an important component in ribosome biogenesis in association with TOR (Honma et al., 2006). Therefore, TOR/insulin pathways may be involved in regulating development and life span in $C$. elegans via NOG-1.To further test NOG-1 involvement in regulating the life span of $C$. elegans, we compared the life span of nog-1 RNAi-treated worms with the life spans of control (gfp), daf-2, and daf-16 knockdown worms. The mean life span of nog-1 knockdown worms was $21.2 \pm 0.40$ days, which was significantly longer (15\%) than that of control RNAi worms (18.46 \pm 0.41 days) (Fig. 4A). However, the extended life span of nog-1 knockdown worms was not as prominent as that the life span of daf-2 RNAi worms ( $29.85 \pm 0.67$ days).

Since daf-16 is a well-known downstream target in the insulin/IGF-1 pathway and is negatively regulated by daf-2, daf-16 RNAi worms showed extremely shortened life spans (12.9 \pm 0.25 days). In order to test if NOG-1 may act in the insulin/IGF1 pathway directly or indirectly, we performed similar experiments under a daf-16 (mu86) background. As expected, there were no significant differences among the three categories of treatment: control (12.21 \pm 0.24 days), daf-2 (13.2 \pm 0.3 days), and nog-1 (12.29 \pm 0.27 days) (Fig. 4B). The results further indicated that daf-16 might be a downstream component of NOG-1-mediated extended life span.

Silent information regulator 2 protein (Sir2 or Sirtuin) is an $\mathrm{NAD}^{+}$-dependent protein deacetylase that has been identified in yeast, flies, and mammals, and recently in $C$. elegans as well (Bamps et al., 2009; Burnett et al., 2011). In C. elegans, Sirtuin gene (sir-2.1) over-expression leads to life span extension and 
A

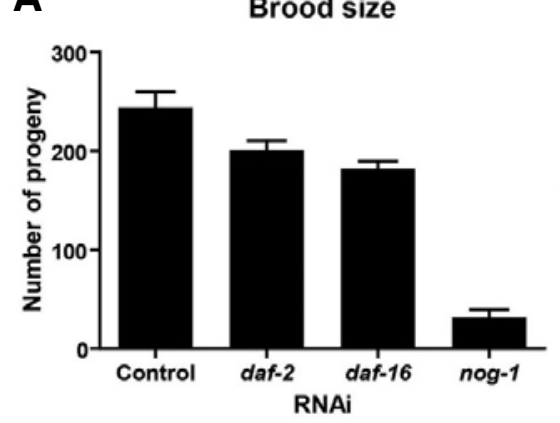

B

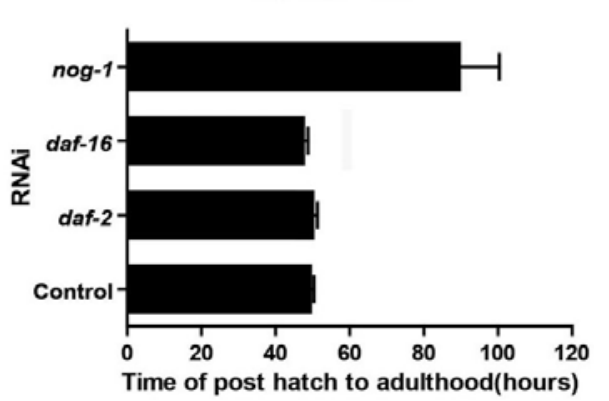

Fig. 3. Brood size and growth rate in nog-1 knockdown animals. (A) Brood sizes of control (gfp RNAi), daf-2, daf16, and nog-1 RNAi treated worms (n $=20$, average of 5 trials). (B) Growth rates of control (gfp RNAi), daf-2, daf16, and nog-1 RNAi treated worms (n $=20$, average of 5 trials).
A

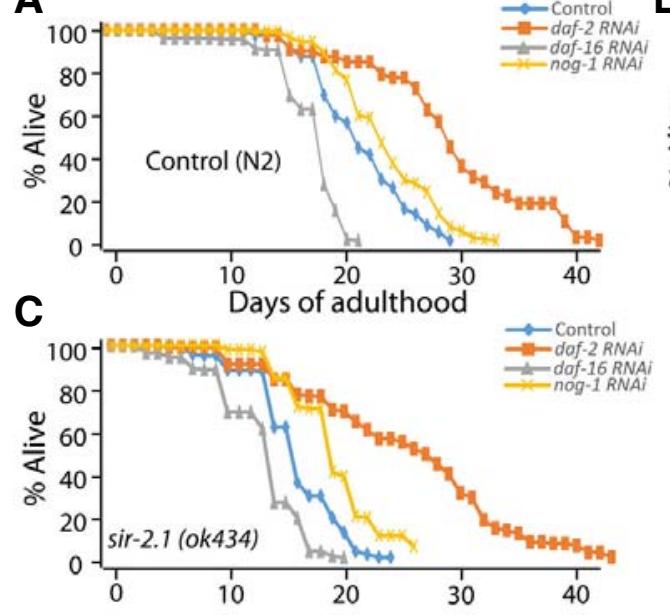

B

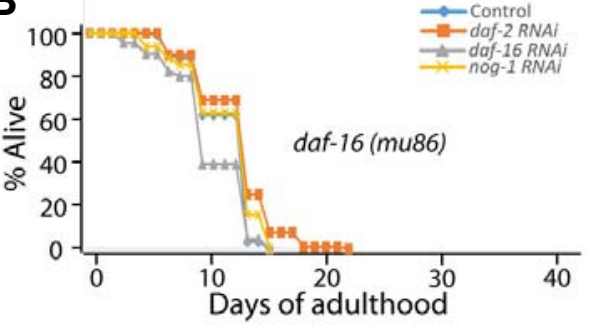

Fig. 4. NOG-1 regulates life span via insulin/IGF-1 pathway. Life span analyses at $20^{\circ} \mathrm{C}$. (A) The average life spans of control (gfp RNAi), daf-2, daf-16 and nog-1 RNAi treated worms in N2 background ( $\mathrm{n}=172,122$, 185 , and 182 respectively). (B) The average life spans in daf16(mu86) background $(n=126$, 119,135 , and 155 respectively). (C) The average life spans in sir-2.1(ok434) $(n=160,151,150$, and 133 respectively). deletion of the gene shortens life span (Viswanathan et al., 2005). To determine whether the Sir-2.1-mediated pathway is involved in regulating life span via NOG-1 in C. elegans, the life span assay was performed under a sir-2.1(ok434) background (Fig. 4C). Even in the sir-2.1(ok434) background, nog-1 RNAitreated worms showed significantly increased life spans compared to the control (gfp RNAi): $19.35 \pm 0.4$ and $15.89 \pm 0.3$ days, respectively. Therefore, NOG-1-mediated life span extension may be independent of the Sir-2.1 pathway.

\section{NOG-1 associates with RLP-24.2 (RIp24p) to affect life span of $C_{\text {. elegans }}$}

NOG-1 is essential for the maturation of 605 ribosome particles. Specifically, it is required for $35 \mathrm{~S}$ rRNA processing, which is an early step of ribosome biosynthesis (Jensen et al., 2005). However, NOG-1 is distributed throughout the nucleus and is also known to associate with a number of 60S RPs. Yeast ribosomal like protein 24 (Rlp24p) encodes a large ribosomal subunit L24 protein; yeast Rlp24 is essential for both early and late stages of 605 ribosome biogenesis and is known to interact with NOG1, both genetically and physically (Harnpicharnchai et al., 2001; Saveanu et al., 2003).

We predicted that over-expressing nog-1 or rlp-24.2 in C. elegans may alter its life span. Thus, we generated nog-1and/or rlp-24.2 over-expression constructs with heat shock promoters (see "Materials and Methods"). Transgenic worms (synchronous L4 stage larvae) or their eggs were given heat shock and subsequently examined for the effects of the over-expression of desired genes in relation to the life span of the worms.

Either nog-1 or rlp-24.2 over-expression from embryos resulted in decreased adult longevity compared to the control ( $g f p$; Fig. 5A). Interestingly nog-1 and rlp-24.2 double over-expression displayed a further decrease in life span. On the other hand, the results from over-expression in L4 stages showed a similar pattern but to a lesser degree (Fig. 5B). Even though the differences between the controls and nog-1 over-expression from L4 were minimal, the cumulative decrease in double overexpression indicates NOG-1 involvement in longevity. In addition, the decline in longevity in nog-1 and $r l p-24.2$ over-expression worms indicates physical and functional interactions between NOG-1 and RLP-24.2. However, nog-1 and rlp-24.2 double RNAi treatment showed no additive increase of longevity (data not shown).

\section{NOG-1 influences fat storage in C. elegans}

C. elegans has recently emerged as a convenient model for studying the in vivo mechanisms of fat storage, which occurs in the worm's intestine (Mak, 2012). Moreover, there are several studies showing that fat storage is related to the conserved insulin/IGF pathway and the mammalian target of rapamycin (mTOR, let-363 in C. elegans) signaling pathway in C. elegans (Ashrafi, 2007; Mukhopadhyay et al., 2005; Yen et al., 2010).

It is well known that $C$. elegans insulin receptor (daf-2) mutants quite often display temperature-sensitive constitutive dauer formation, and also a non-dauer-inducing condition during the availability of adequate food. When daf-2 mutants were 

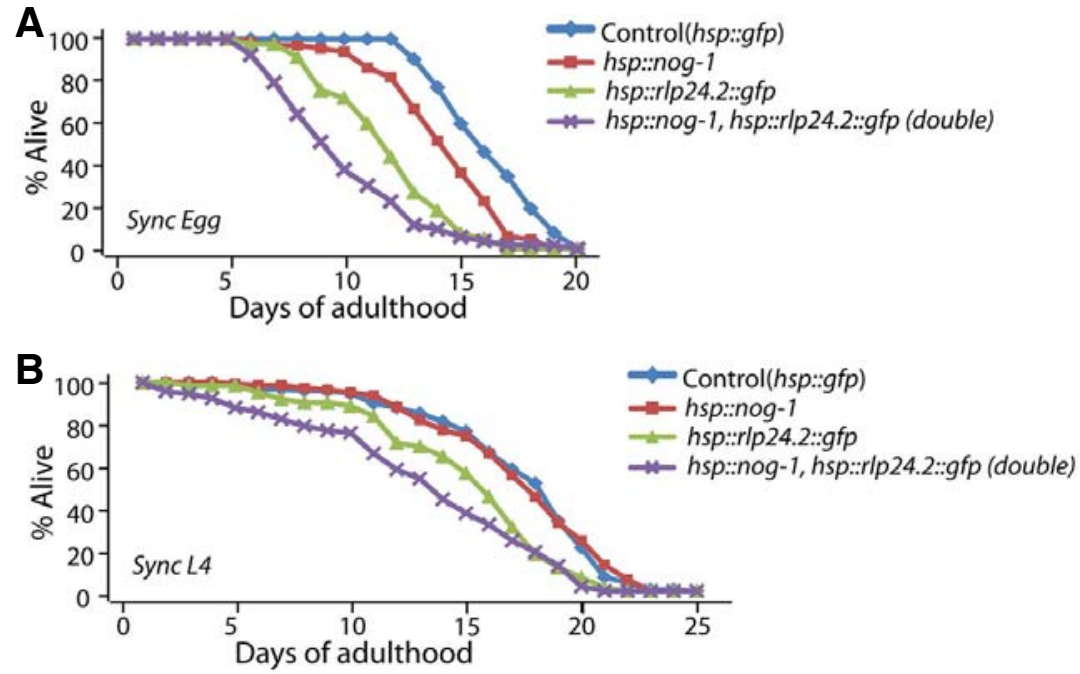

Fig. 5. Over-expression of nog-1. Control (gfp), nog-1, rlp-24.2, and nog-1and rlp-24.2 (double) over-expression constructs with heat shock promoters were used to create transgenic worms (see "Materials and Methods"). The transgenic worms were exposed to heat shock starting from egg stage (A), and from L4 stages (B) continuously. $n=$ approximately 100 .

\section{A} gfp RNAi to $\mathrm{N} 2$

B daf-2 RNAi to $N 2$

C daf-16 RNAi to $\mathrm{N} 2$

D nog-1 RNAi to $N 2$

E nog-1 RNAi to daf-16

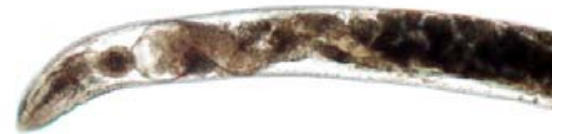

Fig. 6. Fat accumulation assay of nog- 1 knockdown worms. Sudan Black B staining was performed for control ( $g f p, A)$, daf-2 (B), daf-16 (C), nog-1 (D) RNAi treated wild type worms (N2 background), and (E) nog-1 RNAi treatment in daf-16(mu86) mutants.

allowed to grow at permissive temperatures, the adult worm exhibited increased lifespan and fat stores, bypassing the dauer stage (Kenyon et al., 1993; Kimura et al., 1997). These daf-2 mutants further depend on DAF-16, a downstream FOXO transcription factor (Ashrafi et al., 2003; Ogg et al., 1997), for regulating lifespan and development.

Keeping in mind the roles of TOR/insulin pathways in regulating development and life span in worms, as well as fat storage, we therefore hypothesized that NOG-1 might play a role in the mechanism of fat storage in $C$. elegans via the insulin/IGF pathway. In order to examine this, we used a fat soluble dye for staining, Sudan Black B, as a method for visualization of fat storage since Sudan Black B staining is an effective and simple way to reflect the whole fat content in organisms in place of
CARS microscopy (Yen et al., 2010). These results were consistent with those of the life span assays, as Sudan Black B staining experiments revealed increased fat stores in nog-1 knockdown animals that had increased life spans (Fig. 6D) compared to the control RNAi-treated worms (Fig. 6A). This high fat store content was also found in the long-lived daf-2RNAi treated worms (Fig. 6B), whereas daf-16 RNAi-treated worms displayed a relatively decreased fat storage compared to the control RNAi worms, which is an observation consistent with its shorter life span (Fig. 6C).

To further test the role of NOG-1 in fat storage via the insulin/IGF pathway, nog-1 RNAi bacteria were fed to daf-16(mu86) mutants. The nog-1 RNAi/daf-16(mu86) worms (Fig. 6E) showed decreased staining patterns compared to the nog-1 RNAi as well as daf-16 RNAi-treated worms. These results suggest that NOG-1 regulates fat storage in association with life span via the insulin/IGF signaling pathway.

In summary, the nucleolar GTPase NOG-1 in $C$. elegans may have essential functions in growth, development, life span, and fat storage. Specifically, life span and fat storage regulation by NOG-1 are involved via the insulin/IGF pathways.

\section{ACKNOWLEDGMENTS}

Authors gratefully acknowledge Caenorhabditis Genetics Center (CGC) at the University of Minnesota, USA for worm strains and Andrew Fire for the Fire Vectors. The study was supported by research fund from Chosun University, 2008.

\section{REFERENCES}

Ashrafi, K. (2007). Obesity and the regulation of fat metabolism. In WormBook: The Online Review of C. elegans Biology, pp. 1-20.

Ashrafi, K., Chang, F.Y., Watts, J.L., Fraser, A.G., Kamath, R.S. Ahringer, J., and Ruvkun, G. (2003). Genome-wide RNAi analysis of Caenorhabditis elegans fat regulatory genes. Nature 421 , 268-272.

Askjaer, P., Galy, V., Hannak, E., and Mattaj, I.W. (2002). Ran GTPase cycle and importins alpha and beta are essential for spindle formation and nuclear envelope assembly in living Caenorhabditis elegans embryos. Mol. Biol. Cell 13, 4355-4370.

Bamps, S., Wirtz, J., Savory, F.R., Lake, D., and Hope, I.A. (2009) The Caenorhabditis elegans sirtuin gene, sir-2.1, is widely expressed and induced upon caloric restriction. Mech. Age. Dev. 130, 762-770.

Brenner, S. (1974). The genetics of Caenorhabditis elegans. Genetics 
77, 71-94.

Buglino, J., Shen, V., Hakimian, P., and Lima, C.D. (2002), Structural and biochemical analysis of the Obg GTP binding protein. Structure 10, 1581-1592.

Burnett, C., Valentini, S., Cabreiro, F., Goss, M., Somogyvari, M., Piper, M.D., Hoddinott, M., Sutphin, G.L., Leko, V., McElwee, J.J., et al. (2011). Absence of effects of Sir2 overexpression on lifespan in C. elegans and Drosophila. Nature 477, 482-485.

Claypool, J.A., French, S.L., Johzuka, K., Eliason, K., Vu, L., Dodd, J.A., Beyer, A.L., and Nomura, M. (2004). Tor pathway regulates Rrn3p-dependent recruitment of yeast RNA polymerase I to the promoter but does not participate in alteration of the number of active genes. Mol. Biol. Cell 15, 946-956.

Fromont-Racine, M., Senger, B., Saveanu, C., and Fasiolo, F. (2003). Ribosome assembly in eukaryotes. Gene 313, 17-42.

Fuentes, J.L., Datta, K., Sullivan, S.M., Walker, A., and Maddock, J.R. (2007). In vivo functional characterization of the Saccharomyces cerevisiae 60S biogenesis GTPase Nog1. Mol. Genet. Genomics 278, 105-123.

Hannan, K.M., Brandenburger, Y., Jenkins, A., Sharkey, K., Cavanaugh, A., Rothblum, L., Moss, T., Poortinga, G., McArthur, G.A., Pearson, R.B., et al. (2003). mTOR-dependent regulation of ribosomal gene transcription requires S6K1 and is mediated by phosphorylation of the carboxy-terminal activation domain of the nucleolar transcription factor UBF. Mol. Biol. Cell 23, 8862-8877.

Hansen, M., Taubert, S., Crawford, D., Libina, N., Lee, S.J., and Kenyon, C. (2007). Lifespan extension by conditions that inhibit translation in Caenorhabditis elegans. Aging Cell 6, 95-110.

Harnpicharnchai, P., Jakovljevic, J., Horsey, E., Miles, T., Roman, J., Rout, M., Meagher, D., Imai, B., Guo, Y., Brame, C.J., et al. (2001). Composition and functional characterization of yeast $66 \mathrm{~S}$ ribosome assembly intermediates. Mol. Cell 8, 505-515.

Honma, Y., Kitamura, A., Shioda, R., Maruyama, H., Ozaki, K., Oda, Y., Mini, T., Jeno, P., Maki, Y., Yonezawa, K., et al. (2006). TOR regulates late steps of ribosome maturation in the nucleoplasm via Nog1 in response to nutrients. EMBO J. 25, 3832-3842.

Huh, W.K., Falvo, J.V., Gerke, L.C., Carroll, A.S., Howson, R.W., Weissman, J.S., and O'Shea, E.K. (2003). Global analysis of protein localization in budding yeast. Nature 425, 686-691.

Jensen, B.C., Wang, Q., Kifer, C.T., and Parsons, M. (2003). The NOG1 GTP-binding protein is required for biogenesis of the 60 S ribosomal subunit. J. Biol. Chem. 278, 32204-32211.

Jensen, B.C., Brekken, D.L., Randall, A.C., Kifer, C.T., and Parsons, M. (2005). Species specificity in ribosome biogenesis: a nonconserved phosphoprotein is required for formation of the large ribosomal subunit in Trypanosoma brucei. Eukaryotic Cell 4, 3035.

Jia, K., Chen, D., and Riddle, D.L. (2004). The TOR pathway interacts with the insulin signaling pathway to regulate $C$. elegans larval development, metabolism and life span. Development 131, 3897-3906.

Kenyon, C., Chang, J., Gensch, E., Rudner, A., and Tabtiang, R. (1993). A C. elegans mutant that lives twice as long as wild type. Nature 366, 461-464.

Kimura, K.D., Tissenbaum, H.A., Liu, Y., and Ruvkun, G. (1997). daf-2, an insulin receptor-like gene that regulates longevity and diapause in Caenorhabditis elegans. Science 277, 942-946.

Kressler, D., Linder, P., and de La Cruz, J. (1999). Protein transacting factors involved in ribosome biogenesis in Saccharomyces cerevisiae. Mol. Cell. Biol. 19, 7897-7912.

Kutay, U., Lipowsky, G., Izaurralde, E., Bischoff, F.R., Schwarzmaier, P.. Hartmann, E., and Gorlich, D. (1998). Identification of a tRNA-specific nuclear export receptor. Mol. Cell 1, 359-369.

Lafontaine, D.L., and Tollervey, D. (2001). The function and synthesis of ribosomes. Nat. Rev. Mol. Cell. Biol. 2, 514-520.

Lapik, Y.R., Misra, J.M., Lau, L.F., and Pestov, D.G. (2007). Restricting conformational flexibility of the switch II region creates a dominant-inhibitory phenotype in Obg GTPase Nog1. Mol. Cell. Biol. 27, 7735-7744.

Laping, N.J., Olson, B.A., and Zhu, Y. (2001). Identification of a novel nuclear guanosine triphosphate-binding protein differentially expressed in renal disease. J. Am. Soc. Neph. 12, 883-890. Lundquist, E.A. (2006). Small GTPases. In WormBook: The Online Review of $C$. elegans Biology, pp. 1-18.

Mak, H.Y. (2012). Lipid droplets as fat storage organelles in Caenorhabditis elegans: thematic review series: lipid droplet synthesis and metabolism: from yeast to man. J. Lipid Res. 53, 28-33.

Marchler-Bauer, A., Zheng, C., Chitsaz, F., Derbyshire, M.K., Geer, L.Y., Geer, R.C., Gonzales, N.R., Gwadz, M., Hurwitz, D.I., Lanczycki, C.J., et al. (2013). CDD: conserved domains and protein three-dimensional structure. Nucleic Acids Res. 41, D348-352.

Mayer, C., and Grummt, I. (2006). Ribosome biogenesis and cell growth: mTOR coordinates transcription by all three classes of nuclear RNA polymerases. Oncogene 25, 6384-6391.

Mello, C., and Fire, A. (1995). DNA transformation. Methods Cell Biol. $48,451-482$

Moy, T.I., and Silver, P.A. (1999). Nuclear export of the small ribosomal subunit requires the ran-GTPase cycle and certain nucleoporins. Genes Dev. 13, 2118-2133.

Mukhopadhyay, A., Deplancke, B., Walhout, A.J., and Tissenbaum, H.A. (2005). C. elegans tubby regulates life span and fat storage by two independent mechanisms. Cell Metab. 2, 35-42.

Ogg, S., Paradis, S., Gottlieb, S., Patterson, G.I., Lee, L., Tissenbaum, H.A., and Ruvkun, G. (1997). The Fork head transcription factor DAF-16 transduces insulin-like metabolic and longevity signals in C. elegans. Nature 389, 994-999.

Oldham, S., and Hafen, E. (2003). Insulin/IGF and target of rapamycin signaling: a TOR de force in growth control. Trends Cell Biol. 13, 79-85.

Pan, K.Z., Palter, J.E., Rogers, A.N., Olsen, A., Chen, D., Lithgow, G.J., and Kapahi, P. (2007). Inhibition of mRNA translation extends lifespan in Caenorhabditis elegans. Aging Cell 6, 111-119.

Powers, T., and Walter, P. (1999). Regulation of ribosome biogenesis by the rapamycin-sensitive TOR-signaling pathway in Saccharomyces cerevisiae. Mol. Biol. Cell 10, 987-1000.

Saveanu, C., Namane, A., Gleizes, P.E., Lebreton, A., Rousselle, J.C., Noaillac-Depeyre, J., Gas, N., Jacquier, A., and FromontRacine, M. (2003). Sequential protein association with nascent $60 S$ ribosomal particles. Mol. Cell. Biol. 23, 4449-4460.

Schmelzle, T., and Hall, M.N. (2000). TOR, a central controller of cell growth. Cell 103, 253-262.

Steffen, K.K., MacKay, V.L., Kerr, E.O., Tsuchiya, M., Hu, D., Fox L.A., Dang, N., Johnston, E.D., Oakes, J.A., Tchao, B.N., et al. (2008). Yeast life span extension by depletion of 60 s ribosomal subunits is mediated by Gen4. Cell 133, 292-302.

Tsang, C.K., Bertram, P.G., Ai, W., Drenan, R., and Zheng, X.F. (2003). Chromatin-mediated regulation of nucleolar structure and RNA Pol I localization by TOR. EMBO J. 22, 6045-6056

Tschochner, H., and Hurt, E. (2003). Pre-ribosomes on the road from the nucleolus to the cytoplasm. Trends Cell Biol. 13, 255-263.

Urban, A., Neukirchen, S., and Jaeger, K. E. (1997). A rapid and efficient method for site-directed mutagenesis using one-step overlap extension PCR. Nucleic Acids Res. 25, 2227-2228.

Viswanathan, M., Kim, S.K., Berdichevsky, A., and Guarente, L. (2005). A role for SIR-2.1 regulation of ER stress response genes in determining $C$. elegans life span. Dev. Cell 9:605-615.

Venema, J., and Tollervey, D. (1999). Ribosome synthesis in Saccharomyces cerevisiae. Ann. Rev. Genet. 33, 261-311.

Yang, J.S., Nam, H.J., Seo, M., Han, S.K., Choi, Y., Nam, H.G., Lee, S.J., and Kim, S. (2011). OASIS: online application for the survival analysis of lifespan assays performed in aging research PLoS One 6, e23525

Yen, K., Le, T.T., Bansal, A., Narasimhan, S.D., Cheng, J.X., and Tissenbaum, H.A. (2010). A comparative study of fat storage quantitation in nematode Caenorhabditis elegans using label and label-free methods. PLoS One 5, e12810.

Yudin, D., and Fainzilber, M. (2009). Ran on tracks--cytoplasmic roles for a nuclear regulator. J. Cell Sci. 122, 587-593. 